\title{
Sentiment Analysis with Cognitive Attention Supervision
}

\author{
Erik S. McGuire ${ }^{\dagger, *}$, Noriko Tomuro ${ }^{\dagger}$ \\ $\dagger$ DePaul University
}

\begin{abstract}
Neural network-based language models such as BERT (Bidirectional Encoder Representations from Transformers) use attention mechanisms to create contextualized representations of inputs, conceptually analogous to humans reading words in context. For the task of classifying the sentiment of texts, we ask whether BERT's attention can be informed by human cognitive data. During training, we supervise attention with eye-tracking and/or brain imaging data and combine binary sentiment classification loss with these attention losses. We find that attention supervision can be used to manipulate BERT attention to be more similar to the ground truth human data, but that there are no significant differences in sentiment classification accuracy. However, models with cognitive attention supervision more frequently misclassify different samples from the baseline models-they more often make different errors-and the errors from models with supervised attention have a higher ratio of false negatives.
\end{abstract}

Keywords: neural networks, deep learning, transformers, cognitive science, natural language processing, sentiment analysis

\section{Introduction}

To label the sentiment of a text, a human reader may consider the affect (e.g., pleasant or unpleasant) that it evokes, attribute opinions to its author or narrator, or imagine how the text would be received by others, depending on the type of text (e.g., a story or a review) or annotation guidelines [1]. As supervised machine learning approaches have become more prevalent in Natural Language Processing (NLP) the use of crowdsourcing [2] platforms such as Amazon Mechanical Turk to annotate data has been a convenient tool, but it can prove challenging to obtain high quality labels [3]. Additionally, this approach is susceptible to a self-selection bias, where raters who choose to work on a task may share particular characteristics that are not representative of the target population. There may also be a social desirability bias or demand effects, where raters may detect experimenter cues and feel pressured to match expectations [4-6].

Self-reports have been shown to be frequently inaccurate [7], and readers can not effectively recall eye movements [8-10]. The use of objective recordings of language processing behaviors may therefore be complementary to reader annotations as model training data. When reading, reliable patterns of gaze trajectories and brain activity can be recorded and these cognitive linguistic signals can be applied to NLP tasks [11]. The use of eye movements during reading dates to psychological and physiological studies in the late 19th century [12], and brain research has a long legacy of language processing studies, but their use in NLP [13] and machine learning (such as predicting fMRI activity from nouns; [14]) is a more recent phenomenon.

The operative assumption in recent cognitive NLP studies is to leverage the data humans produce during naturalistic reading with neural networks, with the aim of inducing human-like biases and influencing task outcomes. Reading sentences of a particular affective valence, for example, might elicit brain activations or gaze behaviors in readers that can be exploited by models to learn biases that can be generalized to similar inputs. Neural

*emcguir8@depaul.edu

This article is () 2021 by author(s) as listed above. The article is licensed under a Creative Commons Attribution (CC BY 4.0) International license (https://creativecommons.org/licenses/by/4.0/legalcode), except where otherwise indicated with respect to particular material included in the article. The article should be attributed to the author(s) identified above. 
networks can be augmented with cognitive data through a variety of means, such as regularizing attention with eye-tracking [15] and/or electroencephalography (EEG) data [16]. The firing of neuronal populations generate electric potentials along the scalp which can be directly recorded by EEG, and by associating eye movements with performance, eye-tracking (ET) can provide an indirect estimate of attentional focus and cognitive strategies [17]. In this work, we attempt to induce biases in the self-attention weights produced by BERT ${ }^{1}$ [18] during fine-tuning, in order to influence the representations used for binary sentiment classification and alter predictions. To do so, we take a multi-task learning (MTL) approach and combine the main classification loss with auxiliary losses derived from supervising attention weights. To supervise attention, we use ET and EEG data taken from the Zurich Cognitive Language Processing Corpus $\left(\mathrm{ZuCo}^{2} ;[19]\right)$ as the ground truth.

\section{Related work}

Eye-tracking appears to be the more established modality for augmenting models; among the key terms used in gaze behavior studies [20], fixations are the pauses of the eyes to focus on particular areas; saccades are the rapid movements (measured in milliseconds) from one point to another, such as to a later or previous word. Signals from ET and EEG have been combined [11] so that eye-screen positions are not the sole source of inference about language processing (e.g., that unfamiliar words are fixated upon for longer durations). EEG is known to have a high temporal resolution, but it exhibits a lower spatial resolution compared to other neuroimaging techniques due to interference from the scalp [21]. EEG can contribute additional information about language processing through, for example, different amplitudes for event-related potentials (ERPs) corresponding to word frequency [22].

Cognitive NLP tasks that have been studied in recent years include sentiment analysis [23], part-of-speech (POS) tagging [24], and named entity recognition (NER) [25]. Typically for neural network-based approaches, a recurrent architecture such as a bidirectional Long Short-Term Memory (biLSTM) network has been used; more recently a variant of BERT was used for question answering with ET prediction as the auxiliary task [26]. MTL has been used for sentiment analysis and NER with learning gaze behavior as the auxiliary task $[27,28]$, and a combination of gaze and brain data has been applied to a suite of NLP tasks [11], including sentiment analysis, using approaches such as predicting cognitive data or using those data to augment input embeddings. Auxiliary attention loss based on ET [15] or EEG [16] data has been implemented such that a model learns to increase attention to tokens emphasized by human subjects with joint classification and attention losses.

\section{Data}

Table 1. Statistics and stratification for the Stanford Sentiment Treebank (SST-3) dataset of 11,855 samples, and for the ZuCo subset of Stanford Sentiment Treebank. Removing the neutral samples leaves 9,613 samples (SST-2). Filtering samples from SST that are also in $\mathrm{ZuCo}$ for the first stage of fine-tuning leaves 9,350 samples by removing 189 from the train set, 24 from the dev set, and 50 from the test set.

\begin{tabular}{|c|c|c|c|c|c|c|}
\hline Split & negative & neutral & positive & Total (SST-3) & Total (SST-2) & Total (Filtered SST-2) \\
\hline train & 3310 & 1624 & 3610 & 8544 & 6920 & 6731 \\
\hline dev & 428 & 229 & 444 & 1101 & 872 & 848 \\
\hline test & 912 & 389 & 909 & 2210 & 1821 & 1771 \\
\hline Total & 4650 & 2242 & 4963 & 11855 & 9613 & 9350 \\
\hline ZuCo & 140 & 137 & 123 & 400 & 263 & - \\
\hline
\end{tabular}

$1_{\text {https: //huggingface.co/bert-base-uncased }}$

${ }^{2}$ https://osf.io/2urht/ 
For sentiment analysis, we use an English corpus of movie review excerpts, the Stanford Sentiment Treebank (SST) [29]. The dataset and splits (Table 1) are furnished by torchtext $^{3}$, and feature ternary labels (SST-3) by default. Positive and negative classes each take up approximately $40 \%$ of splits. We perform binary sentiment analysis (SST-2) by excluding neutral samples. For the cognitive data we use $\mathrm{ZuCo}$ [19], a corpus of ET and EEG recordings in which 12 adult subjects (fluent English speakers) read sequences at their own speed, with brain recordings synchronized to onsets of eye fixations on words, so that each word in each sentence has corresponding ET and EEG data. The corpus contains 400 review excerpts from SST and 707 biographical sentences from a Wikipedia relation extraction dataset [30]. In this work we select from the ZuCo-SST subset.

For ET, for each word type we had access to five features: number of fixations, first fixation duration (FFD), gaze duration (sum of fixations), total reading time (TRT, the sum of the word's fixations including regressions to it), and go-past time (sum of fixations prior to progressing, including regressions to previous words). In the creation of $\mathrm{ZuCo}$, EEG electrode values were mapped to first-pass fixation onsets to create fixation-related potentials (FRPs), so that each word has 105 values. We use these as EEG features and we average the respective ET and EEG features over all subjects rather than using features from single subjects, as this has been shown to reduce variability of results [21] and mitigate overfitting [31]. To obtain a single ET value for each token, the mean fixation duration (MFD) has been used [15] by dividing the TRT by number of fixations, but in this study we use TRT as a proxy for overall attention to a word in a sentence, as there is no best practice, to our knowledge. We obtain a scalar EEG feature for each word in a sequence by taking the mean [11]; rather than the maximum [16] electrode value.

\section{Method}

In the first stage of fine-tuning, we perform binary sentiment analysis ${ }^{4}$ using SST-2 with the SST samples that are in the ZuCo subset removed (Table 1), as the latter sentences will be used for sentiment analysis and attention supervision in the second stage of fine-tuning. Standard ${ }^{5}$ categorical cross-entropy loss $\mathcal{L}_{C E}$ is calculated for each sentence $j$ in batches of size $M$ with sentence-level predictions for the $C$ classes, $\hat{y} \in \mathbb{R}^{M \times C}$, and a vector of target class indices $t \in \mathbb{Z}^{M}$ where $0 \leq t_{j}<C$.

$$
\mathcal{L}_{C E}(\hat{y}, t)=-\frac{1}{M} \sum_{j}^{M} \ln a_{t_{j}}
$$

where $a_{t_{j}}$ is the $t_{j}$-th value of the softmax of sample $j$ 's $C$ prediction scores $\varphi\left(\hat{y}_{j}\right)$ :

$$
a=\varphi\left(\hat{y}_{j}\right) \quad a_{t_{j}}=\frac{e^{\hat{y}_{j t_{j}}}}{\sum_{k}^{C} e^{\hat{y}_{j k}}}
$$

In the second stage of fine-tuning, we continue with binary sentiment analysis ${ }^{6}$ with additional auxiliary attention losses. For this stage, we use the subset of SST-2 samples that are in the ZuCo data. From a model's attention outputs for a given sentence of $N$ tokens we seek a competitive distribution of $N$ weights to act as an analog to the foci of a human reader. In our implementation, each of BERT's 12 layers uses 12 attention heads, described as representation subspaces from which to attend [32]. Each head produces a

\footnotetext{
${ }^{3}$ https://torchtext.readthedocs.io/en/latest/datasets.html\#sentiment-analysis

${ }^{4}$ Batch size 16, learning rate 5e-5, stopping at 2 epochs when dev accuracy stabilizes as loss increases.

${ }^{5}$ Notation reflects its PyTorch/HuggingFace implementation.

${ }^{6}$ Stopping at 6 epochs (100 steps), when dev set accuracy plateaus while loss continues to increase.
} 
token-token matrix of self-attention weights, and these weights transform the input sentence tokens into context vectors whose features reflect those of the most attended tokens. To influence this transformation of a sequence's token vectors by biasing the weights with $\mathrm{ZuCo}$ data $\alpha^{\prime} \in \mathbb{R}^{1 \times N}$, from these layers' attention matrices $A \in \mathbb{R}^{12 \times 12 \times N \times N}$ we require a single vector of weights $\alpha \in \mathbb{R}^{1 \times N}$ for comparison. To obtain this, we treat the last layer $L$ 's sentence classification token [CLS] as our imaginary reader, as it is conventionally used as the sentence representation for tasks such as classification [18], as well as attention analysis [33]. Consequently, from each matrix we take $A_{L, 1: 12,1,1: N^{-}}$the row of weights accorded to tokens by the [CLS] token-and average the resulting 12 vectors over the heads to obtain a $\alpha \in \mathbb{R}^{1 \times N}$ of model attention weights that [CLS] gives to the sentence tokens. This aggregate is supervised during training: in this way, each representation subspace which informs the context vector ultimately used by the model for classification is independent yet constrained by the same range of human values.

Obtaining human scores for sequence tokens requires preprocessing. As $\mathrm{ZuCo}$ uses a word-level lexicon but BERT uses subword tokenization, in order to find matching entries we first split the $\mathrm{ZuCo}$ words into BERT tokens, evenly dividing values between each subword piece (e.g., when tokenizing "delicacy" $\rightarrow$ ["del", "\#\#ica", "\#\#cy"] in sentence $j$, each piece is allotted a third of the $\mathrm{ZuCo}$ value), a technique previously used by [26]. We pass the human ET and EEG token values $z_{E T}$ and $z_{E E G}$ through a softmax layer to obtain two distributions over sentences, vectors $\alpha_{E T}^{\prime \prime}$ and $\alpha_{E E G}^{\prime}$. Because ET features such as TRT are much larger, measured in milliseconds, than the small EEG microvoltages $(\mu V)$, the raw ET values' softmax output $\alpha_{E T}^{\prime}$ would peakier than $\alpha_{E E G}^{\prime}$, providing an extremely low entropy signal where weights are forced onto one or two tokens. To preserve the original signal, we first reduce each ET token value by dividing by the maximum value for its sentence, returning softmax output $\alpha_{E T}^{\prime \prime}$. After softmax, each sentence consequently has a contextspecific distribution for each modality ET and EEG, reflecting the averaged responses of the human subjects. Following numerous other studies which have performed explicit attention supervision [34-37], we compute two attention losses as the Kullback-Leibler divergence $\left(D_{K L}\right)$ from aggregate model attention weights $\alpha$ to ET and EEG distributions $\alpha_{E T}^{\prime \prime}$ and $\alpha_{E E G}^{\prime}$. We do so for each sentence $j$ in batches of size $M$ for each modality to obtain eye-tracking loss $\mathcal{L}_{E T}$ and EEG loss $\mathcal{L}_{E E G}$. One or both attention losses are added to the categorical cross-entropy loss to give us the overall multi-task fine-tuning loss, $\mathcal{L}_{M T L}$.

$$
\begin{gathered}
\mathcal{L}_{E T}=\frac{1}{M} \sum_{j}^{M} D_{K L}\left(\alpha_{j}^{\prime \prime E T} \| \alpha_{j}\right) \\
\mathcal{L}_{E E G}=\frac{1}{M} \sum_{j}^{M} D_{K L}\left(\alpha_{j}^{\prime E E G} \| \alpha_{j}\right) \\
\mathcal{L}_{M T L}=\mathcal{L}_{C E}+\mathcal{L}_{E T}+\mathcal{L}_{E E G}
\end{gathered}
$$

where the softmax $\alpha_{j}^{\prime \prime E T}$ is obtained from the max-normalized vector of ET token values for sentence $j$ :

$$
\alpha_{j}^{\prime \prime E T}=\varphi\left(\frac{z_{j}^{E T}}{\max \left(z_{j}^{E T}\right)}\right)
$$

\section{Experimental Results}

\subsection{Ablations}

We perform ablations comparing base BERT fined-tuned on SST-2 for five runs with arbitrary random seeds and varying combinations of the cognitive data. The baseline for 
Table 2. 5 run mean of metrics (incl. macro-F1) at 6 epochs. Bold largest, italics lowest.

\begin{tabular}{|c|r|r|r|r|r|r|r|r|r|r|r|r|}
\hline \multirow{2}{*}{ Model } & \multicolumn{9}{|c|}{ Dev } & \multicolumn{6}{|c|}{ Test } \\
\cline { 2 - 12 } & Acc & F1 & P & R & AUC & Loss & Acc & F1 & P & R & AUC & Loss \\
\hline Baseline & $\mathbf{9 0 . 8}$ & $\mathbf{9 0 . 8}$ & $\mathbf{9 0 . 9}$ & $\mathbf{9 0 . 8}$ & $\mathbf{9 0 . 8}$ & 0.53 & 90.3 & 90.3 & $\mathbf{9 0 . 4}$ & 90.3 & 90.3 & 0.53 \\
ET & 90.1 & 90.1 & 90.2 & 90.2 & 90.2 & $\mathbf{0 . 6}$ & 89.6 & 89.6 & 89.7 & 89.6 & 89.6 & $\mathbf{0 . 6}$ \\
EEG & 90.1 & 90.1 & 90.2 & 90.1 & 90.1 & 0.54 & 90.1 & 90.0 & 90.1 & 90.0 & 90.0 & 0.53 \\
ET+EEG & 89.9 & 89.9 & 89.9 & 89.9 & 89.9 & 0.56 & 90.1 & 90.1 & 90.1 & 90.1 & 90.1 & 0.54 \\
Random ET & 90.6 & 90.6 & 90.7 & 90.6 & 90.6 & 0.48 & $\mathbf{9 0 . 4}$ & $\mathbf{9 0 . 4}$ & $\mathbf{9 0 . 4}$ & $\mathbf{9 0 . 4}$ & $\mathbf{9 0 . 4}$ & 0.45 \\
Random EEG & 90.3 & 90.3 & 90.4 & 90.4 & 90.4 & 0.58 & 89.9 & 89.9 & 89.9 & 89.9 & 89.9 & 0.59 \\
Random ET+EEG & $\mathbf{9 0 . 8}$ & $\mathbf{9 0 . 8}$ & 90.8 & $\mathbf{9 0 . 8}$ & $\mathbf{9 0 . 8}$ & 0.49 & $\mathbf{9 0 . 4}$ & $\mathbf{9 0 . 4}$ & $\mathbf{9 0 . 4}$ & $\mathbf{9 0 . 4}$ & $\mathbf{9 0 . 4}$ & 0.45 \\
\hline
\end{tabular}

comparison is the result of the second stage of fine-tuning on the $\mathrm{ZuCo}$ data with only classification loss. For the ET, EEG, and ET+EEG models, we add ET, EEG, or both attention losses, respectively, to the classification loss. To help distinguish the effects of learning regularities in $\mathrm{ZuCo}$ token attention values from the effects of constraining the range of magnitudes as given by the ZuCo values without such patterns, we likewise create random ET, EEG, and ET+EEG models. For the latter, we replace the respective $\mathrm{ZuCo}$ values with values uniformly sampled from the fixed minimum and maximum of the $\mathrm{ZuCo}$ values, to break patterns while maintaining the range of magnitudes. During training we evaluated model predictions on the dev set, and after training, we evaluated the final models on the test set. Table 2 shows evaluation results. Two-sided Pitman's permutation tests [38] were performed on final accuracies for dev and test sets to assess statistical significance, comparing each of the six models against the baseline. Averaging over five runs, there are no statistically significant differences $(p>0.05)$ between baseline vs. the six respective models: $\mathrm{ET}, \mathrm{EEG}, \mathrm{ET}+\mathrm{EEG}$, and random versions thereof.

\subsection{Attention similarity}

Due to the small size of the ZuCo training set, we use all of its samples during fine-tuning. Thus, the dev and test set sentences-which are filtered to exclude ZuCo sentences-do not have corresponding $\mathrm{ZuCo}$ values. Nonetheless, tokens in these sentences can be assigned $\mathrm{ZuCo}$ values with a lexicon ${ }^{7}$ of "type-aggregated" $[11,24]$ cognitive data, where values are averaged over corpus word occurrences to obtain an aggregated value for that word type. We can think of these as corresponding to the typical values that the average reader gave to words as they encountered them in the ZuCo samples. Each word encountered for a given sentence input is assigned its lexicon entry value or 0 for unknown values. Thus, during training context is retained and $\mathrm{ZuCo}$ coverage of tokens in samples is complete (there is a value corresponding to every token) but confined to the ZuCo subset of SST sentences, whereas with the type-aggregated lexicon context is lost and token coverage is incomplete, but distributions can be formed for novel sentences, allowing us to analyze attention similarity for sentences in the dev and test sets.

To measure the similarity of model attentions and human attention in terms of the most attended words, we take inspiration from a behavioral similarity metric [39], in which model attention maps are compared with human attention maps with a binary classification metric, Area Under the Curve (AUC). To determine whether models learn a generalizable bias in attention we create a measurement to assess the token overlap between the most attended tokens in continuous human and model attention vectors, for sentences in the dev and test sets. We look at tokens scored by model attentions after fine-tuning and the scores given by human data. We choose the top- $k$ tokens using a variety of $k$ values to compare for each sentence, and run the models on all splits, using the methods described in $\S 4$ to obtain

${ }^{7}$ https://github.com/DS3Lab/zuco-nlp/tree/master/sentiment-analysis/embedding_data 
Table 3. Top, Middle: Overlapping top- $k$ for model vs. type-aggregated human attentions on the dev and tests. Bottom: Overlap with sample-specific attention from the $\mathrm{ZuCo}$ set. Averaged over 5 runs, bold cells are largest, italics lowest.

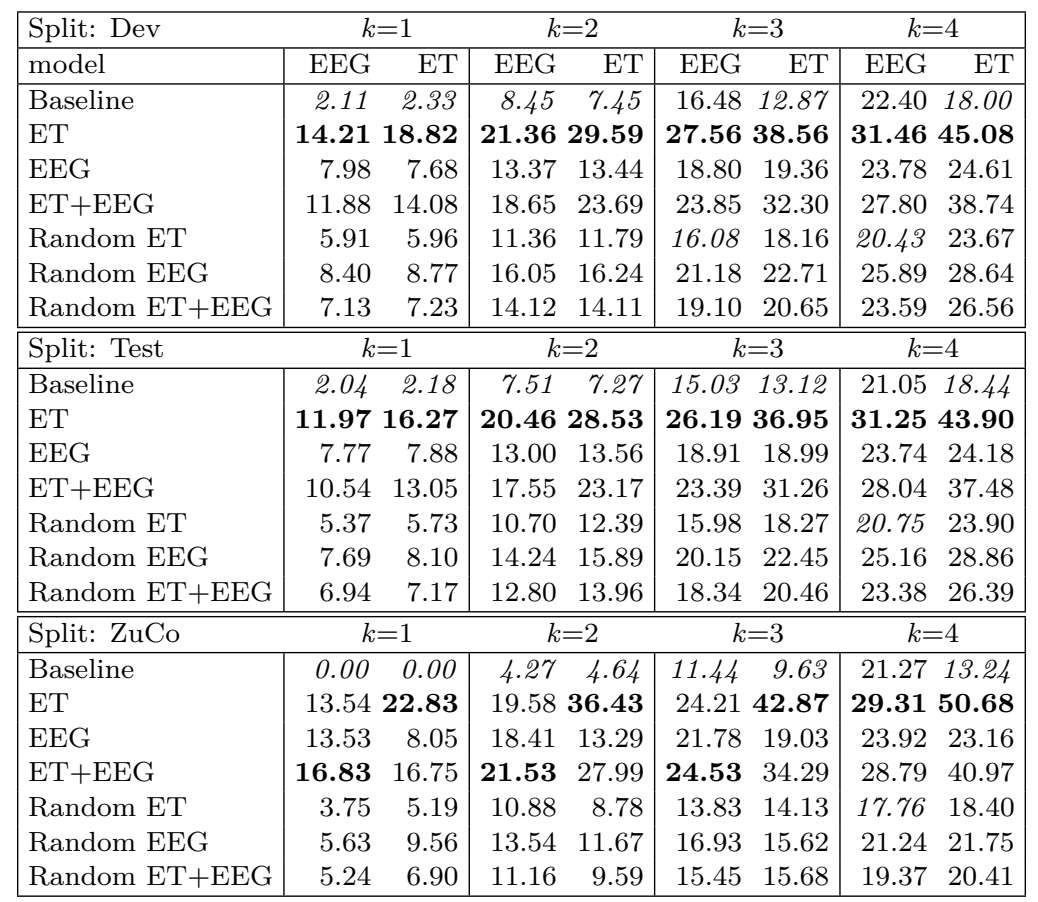

model attentions $\alpha$. We then compute the attention similarity for each dataset by pairwise comparison of each model's attentions with the human data. Results are shown in Table 3. As Equation 5.1 describes, for each model we obtain sets of all samples' token indices and values for the top $k$ attention weights, from both $\alpha$ and $\mathrm{ZuCo}$ values $\alpha^{\prime}$. We then divide the cardinality of the sets' intersection by $k$ to obtain an overlap ratio. To factor in the relative salience of the $k$ weights, we divide their total weight given by the model by their total weight given by $\mathrm{ZuCo}$, and scale the overlap ratio by this percentage, taking the average over each sample $j$ in dataset $D$ :

$$
\operatorname{sim}\left(\alpha_{j}, \alpha_{j}^{\prime}\right)=\frac{1}{D} \sum_{j}^{D}\left[\frac{\left|o_{j}^{k}\right|}{k} \times \min \left(1, \frac{\sum_{i}^{o_{j}^{k}} \alpha_{j i}}{\sum_{i}^{k} \alpha_{j i}^{\prime}}\right)\right]
$$

where $o_{j}^{k}$ is the set of intersecting indices of the top $k$ attention values for sentence $j$ and $\alpha^{\prime}$ corresponds separately to $\alpha^{\prime E E G}$ or $\alpha^{\prime \prime E T}$ (max-normalized $z^{E T}$; Eq. 4.3):

$$
o_{j}^{k}=\alpha_{j}^{k} \cap \alpha_{j}^{\prime k}
$$

As Table 3 shows, the ET model has greatest overlap for both ET and EEG for dev and test sets. While the models were trained with the original sample-specific ZuCo data, the dev and test set overlap is by comparison with the type-aggregated ZuCo lexicon. This lends credence to an association between the type-aggregated and original $\mathrm{ZuCo} \mathrm{ET}$ values, 
corroborating the validity of substituting the former for the latter. However, EEG values seem to lack this association, being higher in overlap only for the ZuCo training set.

\subsection{Error Analysis}

Table 4. MM (mismatches): The percentage of unique errors between model errors and baseline errors out of all errors for both models. $F N$ : Among the unique errors made by a given model vs. the baseline, the percentage of these errors that were false negatives. $F N$ $B$ : Baseline unique errors' percentages of false negatives against the $\mathrm{ZuCo}$ and random models. Bold cells are the highest, italicized lowest.

\begin{tabular}{|l|r|r|r|r|r|r|}
\hline Model & MM (Dev) & MM (Test) & FN (Dev) & FN (Test) & FN B (Dev) vs. & FN B (Test) vs. \\
\hline ET+EEG & 38.97 & $\mathbf{4 3 . 9 6}$ & 57.70 & 66.80 & 32.33 & 29.15 \\
EEG & $\mathbf{4 0 . 9 3}$ & 42.50 & 53.85 & 66.19 & 35.81 & 31.36 \\
ET & 35.70 & 39.66 & $\mathbf{7 0 . 6 6}$ & $\mathbf{7 8 . 9 2}$ & 27.75 & 22.25 \\
Random ET+EEG & 36.62 & 41.82 & 57.50 & 55.00 & $\mathbf{3 8 . 1 4}$ & 34.98 \\
Random EEG & 35.53 & 40.26 & 46.86 & 48.93 & 40.0 & 35.1 \\
Random ET & 35.45 & 39.46 & 59.56 & 68.63 & 33.28 & 30.32 \\
\hline
\end{tabular}

Despite a lack of statistically significant difference in downstream task performance, it is clear that model attentions $\alpha$ are influenced. In order to understand potential consequences of these attentional shifts, where different features may be emphasized in the final sentence representations that BERT uses for classification, we analyze the error types of baseline models by comparison with attention-supervised models. For the dev and test sets, and for each model $\mathcal{M}_{a}$ paired with reference model $\mathcal{M}_{b}$ (e.g., the baseline fine-tuned on SST-2 without attention supervision), we examine mismatches (Equation 5.3): the proportion of the pair's unique errors out of all errors, or the size of the symmetric difference $(\triangle)$ between $\mathcal{M}_{a}$ 's errors $\mathcal{M}_{a}^{\text {inc }}$ and $\mathcal{M}_{b}$ 's errors $\mathcal{M}_{b}^{\text {inc }}$ divided by the size of the union of errors each model makes:

$$
\operatorname{mismatches}\left(\mathcal{M}_{a}, \mathcal{M}_{b}\right)=\frac{\left|\mathcal{M}_{a}^{\text {inc }} \triangle \mathcal{M}_{b}^{\text {inc }}\right|}{\left|\mathcal{M}_{a}^{\text {inc }} \cup \mathcal{M}_{b}^{\text {inc }}\right|}
$$

Table 4 shows mismatches and the number of false negatives (FN) for each model against the baseline on the dev and test splits. By doing so, we note that $\mathrm{ZuCo}$ attention supervision results in more unique errors compared with the baseline than does random attention supervision. We also note that the $\mathrm{ZuCo}$ models-in particular for gaze only supervision evaluated on the test set-have a higher number of false negatives. Notably, the percentage of $\mathcal{M}_{a}$ 's false negative errors is higher among $\mathcal{M}_{a}$ 's mismatches against $\mathcal{M}_{b}$ than the percentage of false negative errors among $\mathcal{M}_{a}$ 's overall errors. It is also evident that random attention supervision of the same tokens-where values are uniformly sampled from the range of $\mathrm{ZuCo}$ values-also increased the number of false negatives, albeit to a lesser extent: as these differences occur by modifying the baseline with human attention supervision, this suggests an influence of regularities in how humans attend to tokens, that is, the existence of patterns in the $\mathrm{ZuCo}$ data that are broken by randomization. We also examine fixes [40]: instances where the baseline model is in error, but the baseline modified with attention supervision is correct. We analyze the percentages of fixes and we also define breaks: when the baseline is correct, but the baseline modified with supervised attention is incorrect. These are shown in Table 5. In contrast to random models, ZuCo-supervised models have a larger number of breaks, and that as with unique errors analyzed previously, more of these breaks were false negatives. Which is to say that modifying the baseline with non-random attention supervision most tends to wrongly flip true positives to negative sentiment predictions. 
Table 5. Fixes/Breaks. Fixes refers to the percentage of all $\mathcal{M}_{b}$ 's errors that $\mathcal{M}_{a}$ correctly predicted. Unique Errors refers to the percentage of all $\mathcal{M}_{a}$ 's errors that $\mathcal{M}_{b}$ correctly predicted. $F N$ here refers to the percentage of all $\mathcal{M}_{a}$ 's errors that are false negative. For the baseline models, averaged dev and test FN percentages are $52.7 \%$ and 43\%. FN (Breaks) is the percentage of all $\mathcal{M}_{a}$ 's errors that were false negative and that $\mathcal{M}_{b}$ predicted correctly. Bold values are largest, italicized the lowest.

\begin{tabular}{|l|r|r|r|r|r|r|r|r|}
\hline & \multicolumn{4}{|c|}{ Dev } & \multicolumn{4}{c|}{ Test } \\
\hline Model & Fixes & Breaks & FN & FN (Breaks) & Fixes & Breaks & FN & FN (Breaks) \\
\hline ET+EEG & 0.21 & 0.28 & 0.60 & 0.17 & $\mathbf{0 . 2 7}$ & 0.29 & 0.55 & 0.20 \\
EEG & 0.24 & $\mathbf{0 . 2 9}$ & 0.59 & 0.17 & 0.25 & $\mathbf{0 . 3 0}$ & 0.55 & 0.21 \\
ET & 0.19 & 0.25 & $\mathbf{0 . 6 3}$ & $\mathbf{0 . 1 8}$ & 0.24 & 0.26 & $\mathbf{0 . 5 8}$ & $\mathbf{0 . 2 1}$ \\
Random ET+EEG & 0.22 & 0.23 & 0.56 & 0.12 & 0.27 & 0.26 & 0.48 & 0.14 \\
Random EEG & 0.21 & 0.22 & 0.53 & 0.10 & 0.26 & 0.25 & 0.46 & 0.12 \\
Random ET & 0.20 & 0.24 & 0.59 & 0.15 & 0.23 & 0.26 & 0.54 & 0.18 \\
\hline
\end{tabular}

\subsection{Input reduction}

Table 6. Statistics for models aggregated over seeds showing false negatives and $\mathrm{ZuCo}$ overlap $(k=4)$ for mismatched errors for dev (top) and test (bottom) sets. FN Congruent refers to the percentage of congruent items which are false negatives. FN EEG Sim and FN ET Sim refer to the proportion of shared words between model and ZuCo values in the top $k$ attentions. Final columns apply the same measurement the baseline which scored the samples correctly while unaffected by the fragment's removal. Bold values are largest, italicized lowest.

\begin{tabular}{|l|r|r|r|r|r|}
\hline Model (dev) & FN Congruent & FN EEG Sim & FN ET Sim & FN EEG B Sim & FN ET B Sim \\
\hline ET+EEG & 53.37 & 57.96 & 66.72 & $\mathbf{3 4 . 0 3}$ & $\mathbf{4 5 . 2 2}$ \\
EEG & 49.68 & 56.56 & 55.89 & 31.22 & 41.44 \\
ET & $\mathbf{7 4 . 7 4}$ & 60.80 & $\mathbf{7 3 . 3 9}$ & 31.99 & 44.08 \\
Random ET+EEG & 51.28 & $\mathbf{7 1 . 6 7}$ & 67.31 & 30.28 & 34.44 \\
Random EEG & 49.78 & 46.33 & 45.17 & 29.33 & 28.67 \\
Random ET & 49.89 & 48.56 & 46.00 & 23.58 & 32.94 \\
\hline Model (test) & FN Congruent & FN EEG Sim & FN ET Sim & FN EEG B Sim & FN ET B Sim \\
\hline ET+EEG & 62.80 & 63.01 & $\mathbf{6 8 . 4 0}$ & 29.09 & 37.39 \\
EEG & 65.43 & 63.44 & 62.49 & 28.11 & 36.66 \\
ET & $\mathbf{7 5 . 0 4}$ & 60.82 & 65.27 & 27.36 & 33.10 \\
Random ET+EEG & 54.12 & $\mathbf{6 5 . 2 1}$ & 63.31 & 34.27 & 40.20 \\
Random EEG & 50.31 & 63.02 & 60.63 & $\mathbf{4 0 . 7 8}$ & $\mathbf{4 4 . 2 1}$ \\
Random ET & 71.80 & 57.23 & 59.46 & 23.67 & 30.58 \\
\hline
\end{tabular}

Input reduction [41] is a method to interpret model errors wherein tokens are systematically removed from an input to discover the minimal version of that input that preserves the original prediction. We create a form of attention-based input reduction and incorporate it into our analysis. Specifically, we explore the erroneous samples by searching for words from the top $k$ attended words and systematically removing them until certain criteria are met, after which we run both the removed words and reduced sentences through the respective models. The criteria are that the removed word must be congruent-obtaining the same sentiment prediction as the original sentence-and that the reduced version of the original sentence formed by removing this congruent word changes $\mathcal{M}_{a}$ 's prediction but not $\mathcal{M}_{b}$ 's prediction. This allows us to discover whether an association can be found between the most attended words and the types of errors made. Choosing $k=4$ as our cut-off, we obtain a pool of instances where removing one or more of the most attended words within the range of $k$ corrects the prediction of model $\mathcal{M}_{a}$ without breaking model $\mathcal{M}_{b}$ 's correct prediction. The results can be seen in Table 6 . For these instances, we observe an especially large number of false negatives for the ET models and an associated high similarity with 
the ET data. While this analysis is not exhaustive, with an arbitrary cut-off at $k=4$, there does appear to be a negativity bias wherein the ZuCo supervision affects model attentions, causing the models to more frequently err by focusing on words that lead to false negative predictions. We plot these samples (see Figure 1) to visualize attention differences. In the bottom plot, "dilithium," a specialized genre-specific word $^{8}$, may be especially noticeable to humans, biasing a model to attend more to that word's pieces ("dil," "\#\#ith," "\#\#ium," their values obtained by splitting the respective ZuCo ET and EEG values for "dilithium") while the baseline model does not to learn to attribute those subword tokens with higher attention.
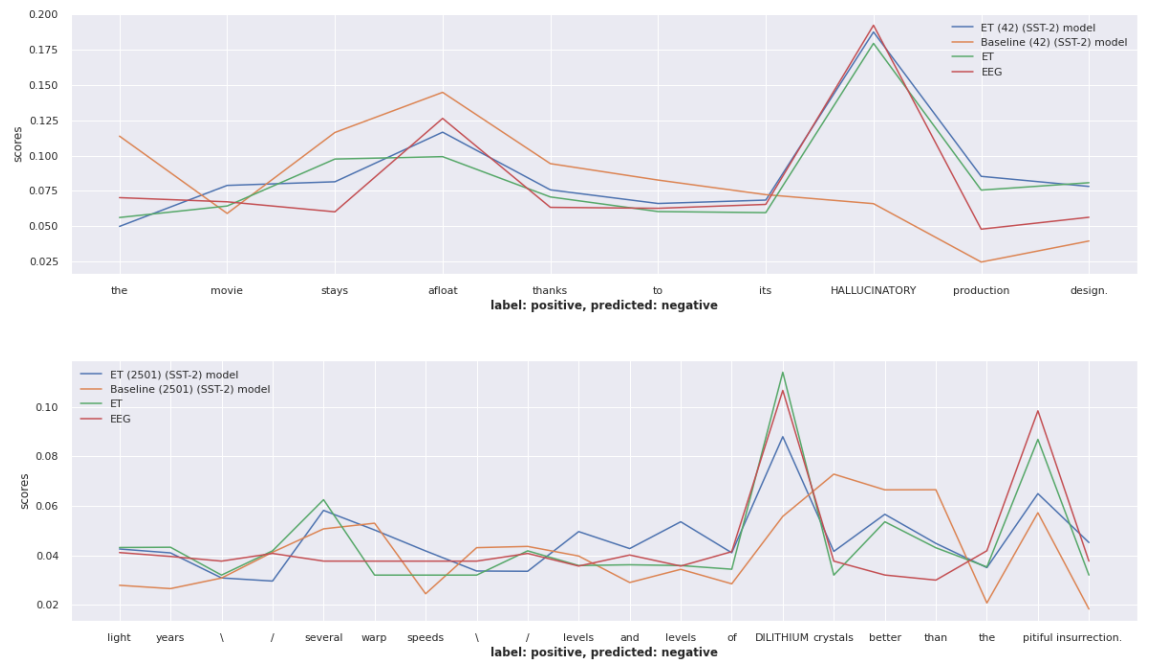

Figure 1. Plots of baseline and attention-supervised model attentions against ZuCo ET and EEG values. In cases like these, regardless of modality or randomness, the attentions are similar across attention-supervised models. Uppercase words are those selected from top $k$ attentions which have a congruent prediction with the sentence's prediction according to the $\mathrm{ZuCo}$ or random $\mathcal{M}_{a}$ and whose removal changes the model's prediction for the remaining sentence while leaving baseline $\mathcal{M}_{b}$ unaffected. The ET model labels "hallucinatory" and the sentence negative, but with "hallucinatory" removed, it labels the remainder positive. The model is induced to attend to "afloat," but the baseline divides its peak attention. Small weights relative to "dilithium" gain prominence when it's removed. We might assume "pitiful" would have a negative influence, but it's counterbalanced by the remaining words and with "dilithium" removed the sentence is predicted positive.

\section{Conclusions}

It appears that BERT fine-tuned on a sentiment classification task converges to similar accuracy despite the application of cognitive attention supervision which shifted aggregated [CLS] attention distributions toward human data. Nonetheless, the errors of attentionsupervised and baseline models consistently differ and this is characterized by more false negatives after attention supervision. Analysis with an input reduction technique indicates an association between the words the $\mathrm{ZuCo}$ models attend to most and these class decisions. By convention, supervised machine learning trains classifiers to correlate with gold standard human judgments using self-reported annotations as training labels. This implicitly or

\footnotetext{
${ }^{8}$ Dilithium is best known as a fictional fuel from the science fiction television show Star Trek.
} 
explicitly sought human-like bias is limited insofar as self-reports can not contain responses which introspect the brain's neuronal activity or the eyes' fixations and saccades. Cognitive data may offer valid judgments, independently of human ground truth labels: a false negative according to a label based on a variety of subjective considerations could be a true negative according to physiological responses. Such cases could reveal nuance glossed over by relying solely on corrective feedback from retrospective annotations. In particular, research has not settled on theories of hardwired universal emotions vs. flexible, context-dependent conceptual constructs [42]. Consequently, it may be useful to maintain a less reductive understanding of affect through cognitive data to avoid assumptions that obsolesce but persist harmfully in systems as scientific consensus evolves.

Other ethical cognitive data concerns that have been described [21] range from loss of privacy to the perpetuation of fossilized human prejudices. Idiosyncratic single-subject models can be created with data from individuals. While intriguing, careful consideration of privacy would be required. In this work, many positive reviews predicted as negative by ZuCo models were comprised of backhanded compliments: these may evoke a primarily negative affect in readers, exposing a conflict or degree of ambiguity. Models informed by non-conscious physiological behavior as a complement to self-reports may therefore more fully describe how humans process language, by integrating both objective and subjective information. To confirm this, future work could employ a trio of annotators to label inputs that engender mismatched predictions between baseline and attention supervised models, investigating which models judges agree with. This work was confined to a small dataset in order to pair each sample with cognitive data from ZuCo. More samples, including from other cognitive corpora, could improve generalization while improving robustness. Future work could also examine out-of-distribution data; Plug and Play Language Models (PPLM) [43] use classifiers as discriminators to control continuations generated by language models. If differences in cognitively-informed behavior obtain across highly novel inputs, generated outputs could be steered by modeling human responses.

\section{References}

[1] S. Mohammad. "A practical guide to sentiment annotation: Challenges and solutions". In: Proceedings of the 7th workshop on computational approaches to subjectivity, sentiment and social media analysis. 2016, pp. 174-179.

[2] N. Stewart, J. Chandler, and G. Paolacci. "Crowdsourcing samples in cognitive science". In: Trends in cognitive sciences 21.10 (2017), pp. 736-748.

[3] J. Ramirez, M. Baez, F. Casati, L. Cernuzzi, and B. Benatallah. "Challenges and strategies for running controlled crowdsourcing experiments". In: arXiv preprint arXiv:2011.02804 (2020).

[4] J. Antin and A. Shaw. "Social desirability bias and self-reports of motivation: a study of Amazon Mechanical Turk in the US and India". In: Proceedings of the SIGCHI Conference on Human Factors in Computing Systems. 2012, pp. 2925-2934.

[5] J. Mummolo and E. Peterson. "Demand effects in survey experiments: An empirical assessment". In: American Political Science Review 113.2 (2019), pp. 517-529.

[6] H. Aguinis, I. Villamor, and R. S. Ramani. MTurk Research: Review and Recommendations. 2020.

[7] E. Vraga, L. Bode, and S. Troller-Renfree. "Beyond self-reports: Using eye tracking to measure topic and style differences in attention to social media content". In: Communication Methods and Measures 10.2-3 (2016), pp. 149-164.

[8] M. L.-H. Vo, A. M. Aizenman, and J. M. Wolfe. "You think you know where you looked? You better look again." In: Journal of Experimental Psychology: Human Perception and Performance 42.10 (2016), p. 1477.

[9] A. D. Clarke, A. Mahon, A. Irvine, and A. R. Hunt. "People are unable to recognize or report on their own eye movements". In: The Quarterly Journal of Experimental Psychology 70.11 (2017), pp. 2251-2270. 
[10] E. M. Kok, A. M. Aizenman, M. L.-H. Võ, and J. M. Wolfe. "Even if I showed you where you looked, remembering where you just looked is hard". In: Journal of Vision 17.12 (2017), pp. 2-2.

[11] N. Hollenstein, M. Barrett, M. Troendle, F. Bigiolli, N. Langer, and C. Zhang. "Advancing NLP with cognitive language processing signals". In: arXiv preprint arXiv:1904.02682 (2019).

[12] N. J. Wade. "Pioneers of eye movement research". In: i-Perception 1.2 (2010), pp. 33-68.

[13] A. Mishra and P. Bhattacharyya. Cognitively Inspired Natural Language Processing: An Investigation Based on Eye-tracking. Springer, 2018.

[14] T. M. Mitchell, S. V. Shinkareva, A. Carlson, K.-M. Chang, V. L. Malave, R. A. Mason, and M. A. Just. "Predicting human brain activity associated with the meanings of nouns". In: science 320.5880 (2008), pp. 1191-1195.

[15] M. Barrett, J. Bingel, N. Hollenstein, M. Rei, and A. Søgaard. "Sequence classification with human attention". In: Proceedings of the 22nd Conference on Computational Natural Language Learning. 2018, pp. 302-312.

[16] L. Muttenthaler, N. Hollenstein, and M. Barrett. "Human brain activity for machine attention". In: arXiv preprint arXiv:2006.05113 (2020).

[17] M. K. Eckstein, B. Guerra-Carrillo, A. T. M. Singley, and S. A. Bunge. "Beyond eye gaze: What else can eyetracking reveal about cognition and cognitive development?" In: Developmental cognitive neuroscience 25 (2017), pp. 69-91.

[18] J. Devlin, M.-W. Chang, K. Lee, and K. Toutanova. "BERT: Pre-training of Deep Bidirectional Transformers for Language Understanding". In: Proceedings of the 2019 Conference of the North American Chapter of the Association for Computational Linguistics: Human Language Technologies, Volume 1 (Long and Short Papers). Association for Computational Linguistics, June 2019, pp. 4171-4186.

[19] N. Hollenstein, J. Rotsztejn, M. Troendle, A. Pedroni, C. Zhang, and N. Langer. "ZuCo, a simultaneous EEG and eye-tracking resource for natural sentence reading". In: Scientific data 5.1 (2018), pp. 1-13.

[20] S. Mathias, D. Kanojia, A. Mishra, and P. Bhattacharya. "A Survey on Using Gaze Behaviour for Natural Language Processing”. In: (July 2020). Ed. by C. Bessiere. Survey track, pp. 49074913.

[21] N. Hollenstein, M. Barrett, and L. Beinborn. "Towards best practices for leveraging human language processing signals for natural language processing". In: Proceedings of the Second Workshop on Linguistic and Neurocognitive Resources. 2020, pp. 15-27.

[22] S. L. Frank, L. J. Otten, G. Galli, and G. Vigliocco. "The ERP response to the amount of information conveyed by words in sentences". In: Brain and language 140 (2015), pp. 1-11.

[23] A. Mishra, D. Kanojia, S. Nagar, K. Dey, and P. Bhattacharyya. "Leveraging Cognitive Features for Sentiment Analysis". In: Proceedings of The 20th SIGNLL Conference on Computational Natural Language Learning. Association for Computational Linguistics, Aug. 2016, pp. $156-166$.

[24] M. Barrett, J. Bingel, F. Keller, and A. Søgaard. "Weakly Supervised Part-of-speech Tagging Using Eye-tracking Data". In: Proceedings of the 54th Annual Meeting of the Association for Computational Linguistics (Volume 2: Short Papers). Association for Computational Linguistics, Aug. 2016, pp. 579-584.

[25] N. Hollenstein and C. Zhang. "Entity Recognition at First Sight: Improving NER with Eye Movement Information". In: Proceedings of the 2019 Conference of the North American Chapter of the Association for Computational Linguistics: Human Language Technologies, Volume 1 (Long and Short Papers). Association for Computational Linguistics, June 2019, pp. 1-10.

[26] J. Malmaud, R. Levy, and Y. Berzak. "Bridging Information-Seeking Human Gaze and Machine Reading Comprehension". In: Proceedings of the 24th Conference on Computational Natural Language Learning. Association for Computational Linguistics, Nov. 2020, pp. 142152.

[27] A. Mishra, S. Tamilselvam, R. Dasgupta, S. Nagar, and K. Dey. "Cognition-Cognizant Sentiment Analysis With Multitask Subjectivity Summarization Based on Annotators' Gaze Behavior". In: Thirty-Second AAAI Conference on Artificial Intelligence. 2018. 
[28] L. G. Bautista and P. Naval. "Towards Learning to Read Like Humans". In: International Conference on Computational Collective Intelligence. Springer. 2020, pp. 779-791.

[29] R. Socher, A. Perelygin, J. Wu, J. Chuang, C. D. Manning, A. Y. Ng, and C. Potts. "Recursive deep models for semantic compositionality over a sentiment treebank". In: Proceedings of the 2013 conference on empirical methods in natural language processing. 2013, pp. 1631-1642.

[30] A. Culotta, A. McCallum, and J. Betz. "Integrating probabilistic extraction models and data mining to discover relations and patterns in text". In: Proceedings of the Human Language Technology Conference of the NAACL, Main Conference. 2006, pp. 296-303.

[31] J. Bingel, M. Barrett, and A. Søgaard. "Extracting token-level signals of syntactic processing from fMRI-with an application to PoS induction". In: Proceedings of the 54th Annual Meeting of the Association for Computational Linguistics (Volume 1: Long Papers). 2016, pp. 747-755.

[32] A. Vaswani, N. Shazeer, N. Parmar, J. Uszkoreit, L. Jones, A. N. Gomez, L. Kaiser, and I. Polosukhin. Attention Is All You Need. 2017. arXiv: 1706.03762 [cs.CL].

[33] K. Clark, U. Khandelwal, O. Levy, and C. D. Manning. "What Does BERT Look at? An Analysis of BERT's Attention". In: Proceedings of the 2019 ACL Workshop BlackboxNLP: Analyzing and Interpreting Neural Networks for NLP. Association for Computational Linguistics, Aug. 2019, pp. 276-286.

[34] L. Qiuxia, S. Khan, Y. Nie, S. Hanqiu, J. Shen, and L. Shao. "Understanding More about Human and Machine Attention in Deep Neural Networks". In: IEEE Transactions on Multimedia (2020).

[35] K. Sharan, A. Ganesan, and T. Oates. "Improving Visual Reasoning with Attention Alignment". In: International Symposium on Visual Computing. Springer. 2019, pp. 219-230.

[36] E. Sood, S. Tannert, D. Frassinelli, A. Bulling, and N. T. Vu. "Interpreting Attention Models with Human Visual Attention in Machine Reading Comprehension". In: Proceedings of the 24th Conference on Computational Natural Language Learning. Association for Computational Linguistics, Nov. 2020, pp. 12-25.

[37] Y. Zhang, J. C. Niebles, and A. Soto. "Interpretable visual question answering by visual grounding from attention supervision mining". In: 2019 IEEE Winter Conference on Applications of Computer Vision (WACV). IEEE. 2019, pp. 349-357.

[38] R. Dror, G. Baumer, S. Shlomov, and R. Reichart. "The hitchhikers guide to testing statistical significance in natural language processing". In: Proceedings of the 56th Annual Meeting of the Association for Computational Linguistics (Volume 1: Long Papers). 2018, pp. 1383-1392.

[39] C. Sen, T. Hartvigsen, B. Yin, X. Kong, and E. Rundensteiner. "Human Attention Maps for Text Classification: Do Humans and Neural Networks Focus on the Same Words?" In: Proceedings of the 58th Annual Meeting of the Association for Computational Linguistics. 2020, pp. 4596-4608.

[40] C. Lin, S. Bethard, D. Dligach, F. Sadeque, G. Savova, and T. A. Miller. "Does BERT need domain adaptation for clinical negation detection?" In: Journal of the American Medical Informatics Association 27.4 (2020), pp. 584-591.

[41] A. Rietzler, S. Stabinger, P. Opitz, and S. Engl. "Adapt or Get Left Behind: Domain Adaptation through BERT Language Model Finetuning for Aspect-Target Sentiment Classification". In: Proceedings of the 12th Language Resources and Evaluation Conference. May 2020, pp. 4933-4941.

[42] M. Gendron and L. F. Barrett. "Emotion perception as conceptual synchrony". In: Emotion Review 10.2 (2018), pp. 101-110.

[43] S. Dathathri, A. Madotto, J. Lan, J. Hung, E. Frank, P. Molino, J. Yosinski, and R. Liu. "Plug and play language models: A simple approach to controlled text generation". In: arXiv preprint arXiv:1912.02164 (2019). 